\title{
Studies of $\Lambda n$ interaction through polarization observables for final-state interactions in exclusive $\Lambda$ photoproduction off the deuteron
}

\author{
Yordanka llieva ${ }^{* \dagger}$ \\ University of South Carolina \\ E-mail: ilievaesc.edu \\ Tongtong Cao \\ University of South Carolina \\ E-mail: caotemail.sc.edu \\ Nicholas Zachariou \\ University of Edinburgh \\ E-mail: nicholasejlab.org
}

\section{The CLAS Collaboration}

Theoretical studies suggest that experimental observables for hyperon production reactions can place stringent constraints on the free parameters of hyperon-nucleon potentials, which are critical for the understanding of hypernuclear matter and neutron stars. Here we present preliminary experimental results for the polarization observables $\Sigma, P_{y}, O_{x}, O_{z}, C_{x}$, and $C_{z}$ for final-state interactions (FSI) in exclusive $\Lambda$ photoproduction off the deuteron. The observables were obtained from data collected during the E06-103 (g13) experiment with the CEBAF Large Acceptance Spectrometer (CLAS) in Hall B at Jefferson Lab. The g13 experiment ran with unpolarized deuteron target and circularly- and linearly-polarized photon beams with energies between 0.5 $\mathrm{GeV}$ and $2.5 \mathrm{GeV}$ and collected about $5 \times 10^{10}$ events with multiple charged particles in the final state. To select the reaction of interest, the $K^{+}$and the $\Lambda$ decay products, a proton and a negative pion, were detected in the CLAS. The missing-mass technique was used to identify exclusive hyperon photoproduction events. Final-state interaction events were selected by requesting that the reconstructed neutron has a momentum larger than $200 \mathrm{MeV} / c$. The large statistics of E06-103 provided statistically meaningful FSI event samples, which allow for the extraction of one- and two-fold differential single- and double-polarization observables. Here we present preliminary results for a set of six observables for photon energies between $0.9 \mathrm{GeV}$ and $2.3 \mathrm{GeV}$ and for several kinematic variables in the $\Lambda n$ center-of-mass frame. Our results are the very first estimates of polarization observables for FSI in hyperon photoproduction and will be used to constrain the free parameters of hyperon-nucleon potentials.

The 8th International Workshop on Chiral Dynamics

29 June 2015 - 03 July 2015

Pisa, Italy

* Speaker.

${ }^{\dagger}$ This work is supported in part by the U.S. National Science Foundation under grant PHY-125782. 


\section{Introduction}

Exclusive hyperon photoproduction off deuteron is an interesting tool to study $Y N$ interactions and to constrain the $Y N$ low-energy scattering parameters. The feasibility of using unpolarized and polarized observables of the reactions $\vec{\gamma} d \rightarrow K \vec{\Lambda} N$ to differentiate among different $Y N$ potential models and to determine the $Y N$ low-energy scattering parameters has been studied in a large number of publications [1-11]. Specifically, the model of [6,7] predicts polarization-observable sensitivity of up to $10 \%$, depending on kinematics, to $\Lambda N$ rescattering and to various $Y N$ potentials. A formalism relating the scattering length for elastic $\Lambda N$ scattering directly to the differential cross section of $\Lambda N$ hadro- and photo-production reactions has been developed in [11]. In this approach, a dispersion integral is derived that allows to relate $\Lambda N$ invariant-mass spectra to the scattering length. The method has been successfully applied to hadroproduction data $p p \rightarrow K^{+} \Lambda p$, where a scattering length of $(1.5 \pm 0.15 \pm 0.3)$ fm was extracted. An analysis of the application of the formalism to observables for $\gamma d \rightarrow K^{+} \Lambda n$ shows that one could extract a spin-averaged $\Lambda n$ scattering length from data below the $\Sigma n$ threshold with theoretical uncertainty of $0.3 \mathrm{fm}$, which is comparable to the uncertainty obtained in the study of hadroproduction data [10].

In a simple picture, the reaction $\gamma d \rightarrow K^{+} \Lambda n$ can be presented in terms of elementary twobody-to-two-body mechanisms. The dominant mechanism is the quasi-free production (QF), in which the kaon and the hyperon are produced off the bound proton and the neutron acts as a spectator (see Fig. 1a). Other contributions come from two-step mechanisms (or final-state interactions, FSI), in which one of the particles produced on one target nucleon then scatters off the other nucleon, as depicted in Fig. 1. Among these final-state interactions, the $\Lambda$ rescattering (diagram (e) on Fig. 1) is of interest for $Y N$ studies as it allows access to the $\Lambda n$ elastic scattering amplitude. In this mechanism, the $\gamma p \rightarrow K^{+} \Lambda$ reaction, producing the hyperon in the first step, is followed by $\Lambda n$ elastic scattering. Other FSI include kaon rescattering (Fig. 1b), pion-mediated (Fig. 1c), and sigma-mediated (Fig. 1d) production and constitute backgrounds for the $Y N$ study. Extracting

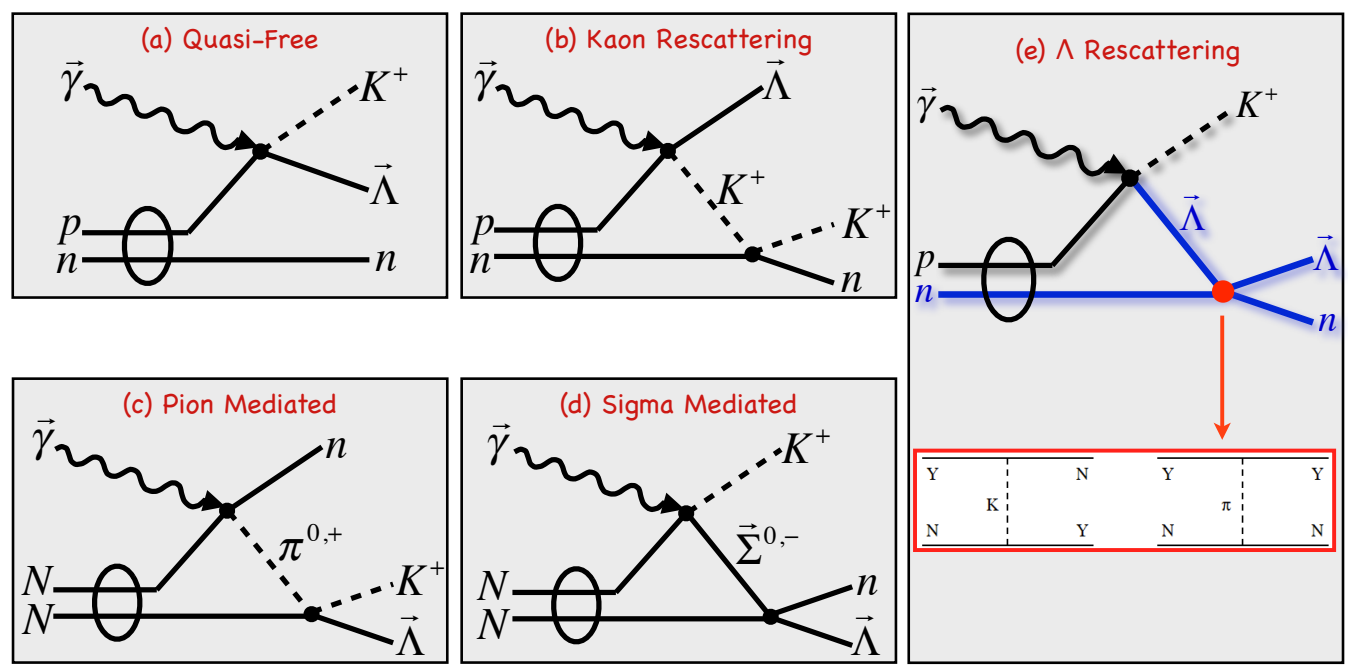

Figure 1: Elementary mechanisms of the reaction $\gamma d \rightarrow K^{+} \Lambda n$. 
information about the $Y N$ scattering amplitude from $\gamma d \rightarrow K^{+} \Lambda n$ data, thus, requires theoretical modeling of the background mechanisms and experimental coverage of a broad kinematic range that can be explored in detail to identify kinematics where the $\Lambda$ rescattering dominates. Estimates of the low-energy $Y N$-potential parameters can be obtained from model fits to the data. The theoretical uncertainties of these parameters can be significantly reduced if a large set of $\gamma d \rightarrow K^{+} \Lambda n$ observables is included in the fits. Here, we will discuss only polarization observables.

For unpolarized target, at a given photon energy, $E_{\gamma}$, the polarized differential cross section for $K Y$ photoproduction off the free nucleon has the form

$$
\begin{aligned}
\frac{d \sigma}{d \Omega}=\frac{d \sigma}{d \Omega}{ }_{0}(1 & -P_{\text {lin }} \Sigma \cos 2 \phi+\alpha \cos \theta_{x}\left(-P_{\text {lin }} O_{x} \sin 2 \phi-P_{\text {circ }} C_{x}\right)-\alpha \cos \theta_{y}\left(-P+P_{\text {lin }} T \cos 2 \phi\right) \\
& \left.-\alpha \cos \theta_{z}\left(P_{\text {lin }} O_{z} \sin 2 \phi+P_{\text {circ }} C_{z}\right)\right),
\end{aligned}
$$

where $d \sigma / d \Omega_{0}$ is the unpolarized cross section, $P_{\text {lin }}$ and $P_{\text {circ }}$ denote the linear and the circular polarization of the photon beam, respectively; $\phi$ is the angle between the photon linear-polarization vector and the reaction plane (see Fig. 2); $\alpha$ is the self-analyzing power of the $\Lambda$ hyperon; $\cos \theta_{x}$, $\cos \theta_{y}$, and $\cos \theta_{z}$ denote the direction cosines of the three-momentum vector of the decay proton in the rest frame of $\Lambda$ (see Fig. 2), and $\Sigma, P, T, O_{x}, O_{z} C_{x}$, and $C_{z}$ are the polarization observables of interest: beam-spin asymmetry, $\Lambda$ induced polarization, target asymmetry, and polarization transfers from linearly- and circrularly-polarized photons to the hyperon, respectively. The expression

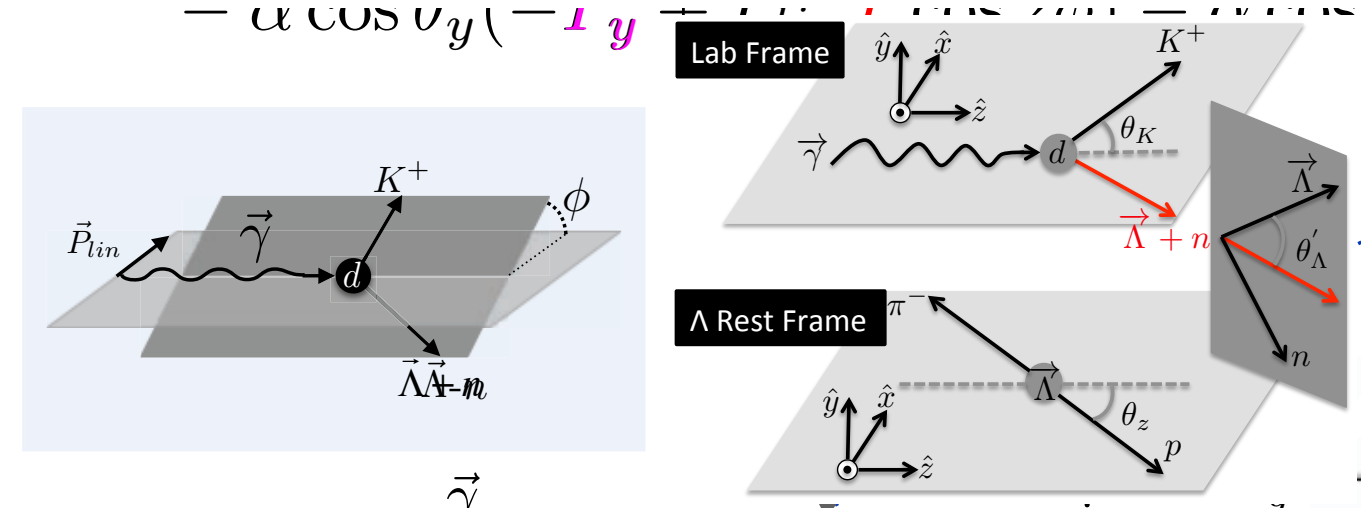

Figure 2: Left: The azimutal angle $\phi$ used in Eq. (1.1) is the angle between the photon linear-polarization vector and the reaction plane. Right: Definition of a Cartesian Reference Plane for the extraction of the polarization observables described in Eq. (1.1). The $Z$ axis points in the direction of the incoming photon. The $Y$ axis is in the direction of the vector product of the photon and the kaon 3-vectors. The $X$ axis complements $Z$ and $Y$ to a right-handed reference frame. The angles $\theta_{x}, \theta_{y}$, and $\theta_{z}$ are defined in the rest frame of $\Lambda$. The angle $\theta_{\Lambda}^{\prime}$ is the angle between the 3-vector of $\Lambda$ and the 3-momentum-vector transfer to the Lambda-neutron system in the lab frame.

of the polarized cross section for the reaction $\gamma d \rightarrow K^{+} \Lambda n$ is more complicated than Eq. (1.1) due to the three-particle final state. More observables, such as helicity asymmetries, can be determined. Here, we focus only on the observables shown in Eq. (1.1).

The objective of our study is to extract a large set of polarization observables for final-state interactions for the reaction $\vec{\gamma} d \rightarrow K^{+} \vec{\Lambda} n$, which can be used to constrain the $\Lambda n$ scattering amplitude and, therefore, the free parameters of $Y N$ potentials. 


\section{Experiment and Data}

The data were collected with the CLAS in Hall B at Jefferson Lab during the E06-103 (g13) experiment [12]. A tagged real-photon beam was incident on a 40-cm-long liquid deuterium target situated $40 \mathrm{~cm}$ upstream from the center of the CLAS. Both, circularly- and linearly-polarized photon beams were used. The circularly-polarized photon beam was produced via the bremsstrahlung technique by impinging a polarized electron beam, delivered by the accelerator, on a thin gold radiator. The polarization of the electrons varied between $80 \%$ and $85 \%$. The direction of the electron polarization was flipped and recorded periodically, which allowed to reduce acceptance effects in the extracted polarization observables. The energy and the polarization of the circularly-polarized photons were in the ranges $0.5 \mathrm{GeV}-2.5 \mathrm{GeV}$ and $30 \%-80 \%$, respectively. The linearly-polarized photon beam was produced by coherent bremsstrahlung by impinging electrons on a $50-\mu \mathrm{m}$ thin diamond radiator. Data were taken at coherent-edge positions of $1.1 \mathrm{GeV}, 1.3 \mathrm{GeV}, 1.5 \mathrm{GeV}, 1.7$ $\mathrm{GeV}, 1.9 \mathrm{GeV}, 2.1 \mathrm{GeV}$, and $2.3 \mathrm{GeV}$. The average photon polarization was $75 \%$. In order to reduce detector acceptance effects in the extracted observables, data were taken at two orientations of the photon polarization vector - parallel and perpendicular to the Hall-B floor. These orientations were regularly changed throughout the experiment by rotating the diamond radiator. In order to ensure a clean real-photon beam incident on the target, the primary electron beam was bent away from the beam line by a dipole magnet that was part of the Hall-B tagger [13]. The latter was also used to determine the energy and the time of the photon that initiated the reaction in the deuteron target.

The final-state particles produced in nuclear reactions were detected by the CLAS [14]. The CLAS was a large-acceptance detector optimized for multi-charged particle detection and was in operation between 1997 and 2012. It consisted of a toroidal magnetic spectrometer, drift chambers, scintillation detectors for timing, electromagnetic calorimeter, and a Cherenkov detector. The toroidal field was created by six superconducting coils mounted symmetrically around the beam line. In the toroidal field, charged particles originating from the target were bent outward or inward relative to the beam line, depending on the sign of their charge. During g13, negatively-charged particles were outbending. The trajectory of charged particles were reconstructed by multi-layer drift chambers that occupied the space between the coils. The reaction vertex was reconstructed by extrapolating the tracks back to the target. Two scintillation detectors measured timing; the Start Counter (ST) and the Time-Of-Flight Detector (TOF). Each of them consisted of scintillation bars, mounted symmetrically around the beam line. The former was situated very near the target, outside of the magnetic field, while the latter was placed behind the drift chambers. The purpose of ST was to determine the start time of the event, while TOF provided a time-of-flight measurement. Thus, for each track the CLAS provided estimates of the track's vertex, magnitude of 3-momentum, path length from the vertex to TOF, vertex time, and time of flight from the vertex to the TOF. These allowed to determine the speed, $\beta$ (in units of $c$ ), for each particle. The Hall-B tagger provided estimates of the energy and timing of each photon incident on the target.

The reaction of interest in this study is $\gamma d \rightarrow K^{+} \Lambda n$. While a significant amount of $K^{+}$makes it through the entire detector before decaying, the hyperon decays mostly in the target, and is reconstructed by detecting its charged decay products, $p$ and $\pi^{-}$. Thus, to select a suitable data subsample, events with only one negatively- and two-positively charged tracks were processed for further analysis. 
In order to identify the particle that has produced a given track, the speed $\beta$ for that track was compared to the expected speed $\beta_{0}$ for a given mass hypothesis. The difference $\Delta \beta=\beta-\beta_{0}$ was constrained to exclude invalid hypotheses. The two positively-charged tracks were each tested against a pion and a proton hypothesis, while the negatively-charged track was tested only against a pion hypothesis. Other selection criteria removed accidental photons, events not originating from the target, and $p \pi^{-}$pairs not produced by a $\Lambda$ decay. The application of these selection cuts yields a reduced data sample containing predominantly $\gamma d \rightarrow K^{+} \Lambda X$ events. The mass, $M_{X}$, and the momentum, $\vec{p}_{X}$, of the missing state were reconstructed by applying four-momentum-vector conservation:

$$
\begin{aligned}
M_{X} & =\sqrt{\left(\tilde{p}_{\gamma}^{2}+\tilde{p}_{d}^{2}-\tilde{p}_{K^{+}}^{2}-\tilde{p}_{\pi^{-}}^{2}-\tilde{p}_{p}^{2}\right)^{2}} \\
\vec{p}_{X} & =\vec{p}_{\gamma}-\vec{p}_{K^{+}}-\vec{p}_{\pi^{-}}-\vec{p}_{p},
\end{aligned}
$$

where $\tilde{p}_{\gamma}, \tilde{p}_{d}, \tilde{p}_{K^{+}}, \tilde{p}_{\pi^{-}}$, and $\tilde{p}_{p}$ denote the 4-vectors of the photon, deuteron, kaon, pion, and proton, respectively.
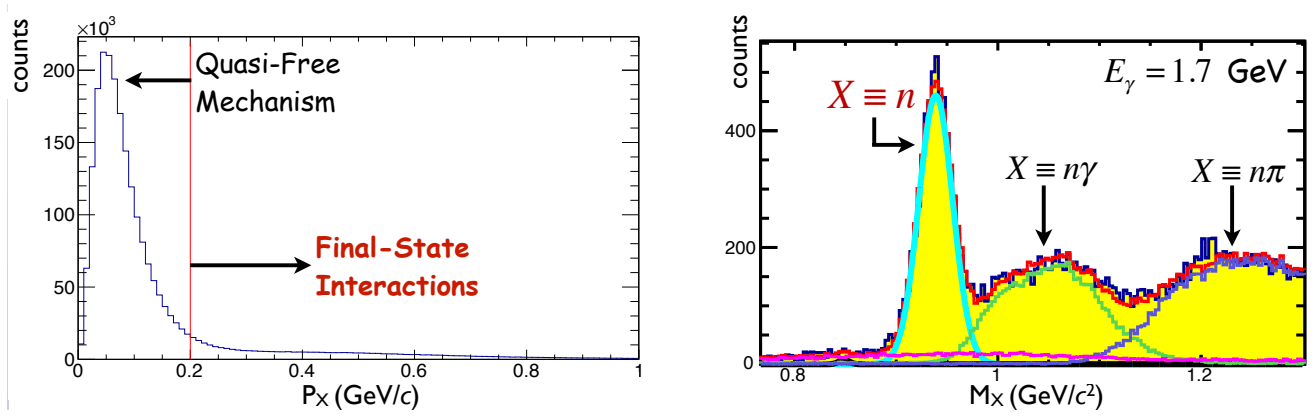

Figure 3: Left: Event distribution over $p_{X}$. For most of the events $p_{X}$ is small, which is consistent with quasi-free production. FSI events are mostly contained in the high-momentum tail of the distribution. One can see that the amount of events in the tail is much smaller than the quasi-free events. The large statistics of g13 makes it possible to extract statistically meaningful observables for FSI events. To reduce the amount of quasi-free events in the data sample, only events with $p_{X}>0.2 \mathrm{GeV} / c$ were processed for further analysis. Right: Event distribution over $M_{X}$ for FSI $\gamma d \rightarrow K^{+} \Lambda X$ events for one energy bin in photon energy. The peak centered around the nominal neutron mass contains the events of interest, while the two structures at higher $M_{X}$ contain predominantly $\Sigma^{0}$ and $\Sigma^{*}$ events, respectively. The solid magenta, green, and violet lines show the distribution of accidental, $\Sigma^{0}$, and $\Sigma^{*}$ background, respectively.

Events produced by the quasi-free mechanism shown in Fig. 1 dominate the reduced data sample. However, the amount of these events can be significantly reduced by requiring the momentum $p_{X}$ to be larger than the typical nucleon Fermi momentum in the deuteron. In our analysis, we process for further analysis only events for which $p_{X}>0.2 \mathrm{GeV} / c$ (see Fig. 3 left). The final step of the selection procedure was the removal of physical and accidental background as shown in the right plot of Fig. 3. The shape of the accidental background was obtained by event-mixing of real data, while the shapes of the $\Sigma^{0}$ and $\Sigma^{*}$ backgrounds were obtained from simulations.

\section{Preliminary Results and Discussion}

In order to extract relevant physics information about $\Lambda n$ scattering from our $\gamma d \rightarrow K^{+} \Lambda n$ FSI 
sample, we need to choose proper kinematic variables for binning. For a given photon energy, the reaction of interest can be described by 5 independent kinematic variables, which can be chosen arbitrarily. Our specific choice of variables is dictated by the kinematics of quasi-free $\gamma p \rightarrow K^{+} \Lambda$ production and of $\Lambda n \rightarrow \Lambda n$ elastic scattering. Based on this consideration, we chose to bin the data in $E_{\gamma}, p_{K^{+}}, \theta_{K^{+}}, W_{\Lambda n} \equiv I M_{\Lambda n}$, and $\theta_{\Lambda}^{\prime}$, where $W_{\Lambda n} \equiv I M_{\Lambda n}$ denotes the square of the total energy in the center-of-mass of the $\Lambda n$ system. Due to limited statistics, we could not bin the data in all the five variables. We extracted one-fold, two-fold, three-fold, and four-fold differential observables.

The beam-spin asymmetry, $\Sigma$, extracted from data taken with linearly-polarized photons, is a single-polarization observable that is of special interest for two reasons. First, it is our smalleststatistical-uncertainty observable. Second, it entirely depends on the photon polarization, and therefore on the physics of quasi-free production in the first step of the reaction. FSI only redistribute the yields from the first step and dilute the beam-spin asymmetry. By definition, $\Sigma$ characterizes the magnitude of the polarized-yield modulation in terms of the azimutal angle $\phi$ between the photon polarization vector $\vec{P}_{\text {lin }}$ and the reaction plane, as shown in Fig. 2. For a two-body to two-body reaction, the angle $\phi$ is uniquely determined, however for a three-body final state, $\phi$ is not unique and there are several possibilities. For instance, one can extract $\Sigma$ from event distributions over $\phi_{n}, \phi_{K}$, or $\phi_{\Lambda}$, which is the azimutal angle between $\vec{P}_{l i n}$ and $\vec{p}_{n}, \vec{p}_{K}$, or $\vec{p}_{\Lambda}$, respectively. Let us denote the corresponding beam-spin asymmetries as $\Sigma_{n}, \Sigma_{K}$, and $\Sigma_{\Lambda}$, respectively. When two final-state particles originate from the same vertex, such as $\Lambda$ and $n$ from $\Lambda n$ scattering, their yields are correlated and the corresponding beam-spin asymmetries should be consistent with each other, i.e., $\Sigma_{\Lambda}$ should be consistent with $\Sigma_{n}$. When $\Lambda$ and $K^{+}$are produced via the pion-mediated mechanism, then $\Sigma_{\Lambda}$ and $\Sigma_{K}$ should be consistent with each other. Thus, the beam-spin asymmetry can be used to probe various kinematics for dominance of specific FSI mechanisms in a model-independent way. Our preliminary results for $\Sigma_{n}, \Sigma_{K}$, and $\Sigma_{\Lambda}$ are shown in Fig. 4. Given the indication, that $\Lambda n$ scattering may be dominant at large $\theta_{\Lambda}^{\prime}$, additional studies will focus on extracting observables specifically at this kinematics. Figure 5 shows one-fold differential estimates of $\Sigma_{K}$ and the polarization transfers $O_{x}$ and $O_{z}$, as functions of $E_{\gamma}, \theta_{\Lambda}^{\prime}, p_{K}$, and $I M_{\Lambda n}$. The three observables were extracted simultaneously from maximum log-likelihood fits to the data. While the beam-spin asymmetry is relatively large and negative at all kinematics, the polarization transfer along the photon direction seems to be almost zero. The polarization transfer $O_{x}$ is mostly positive and significantly non-zero. Overall, the three observables show very little $E_{\gamma}$ and $\theta_{\Lambda}^{\prime}$ dependence when integrated over all other kinematic variables. $\Sigma_{K}$ becomes more negative as $p_{K}$ increases and reaches -0.7 at $p_{K}=1.75 \mathrm{GeV} / c$. The dependence of $\Sigma_{K}$ on the $\Lambda n$ invariant mass suggests that the observable increases in magnitude up to the $\Sigma n$ threshold, where it reaches a value of -0.5 , and decreases in magnitude at higher $\Lambda n$ invariant masses. Fits to the data below the $\Sigma n$ threshold will be done in order to extract an estimate of a spin-averaged $\Lambda n$ scattering length. For a separation of the ${ }^{1} S_{0}$ and ${ }^{3} S_{1}$ scattering lengths, data with polarized deuteron target are needed.

Preliminary one-fold differential estimates for the polarization transfers from circularly-polarized photons to the final-state $\Lambda$ for quasi-free production and FSI are shown in Fig. 6 . As with the beam-spin asymmetry, the polarization transfers $C_{x}$ and $C_{z}$ for FSI show very weak dependence on $E_{\gamma}$ when they are integrated over all other kinematic variables. Thus, to maximize statistics, we will focus on extracting the observables in bins of the other kinematic variables and will integrate over $E_{\gamma}$. These two observables can also be used to probe in more detail the FSI dynamics, espe- 

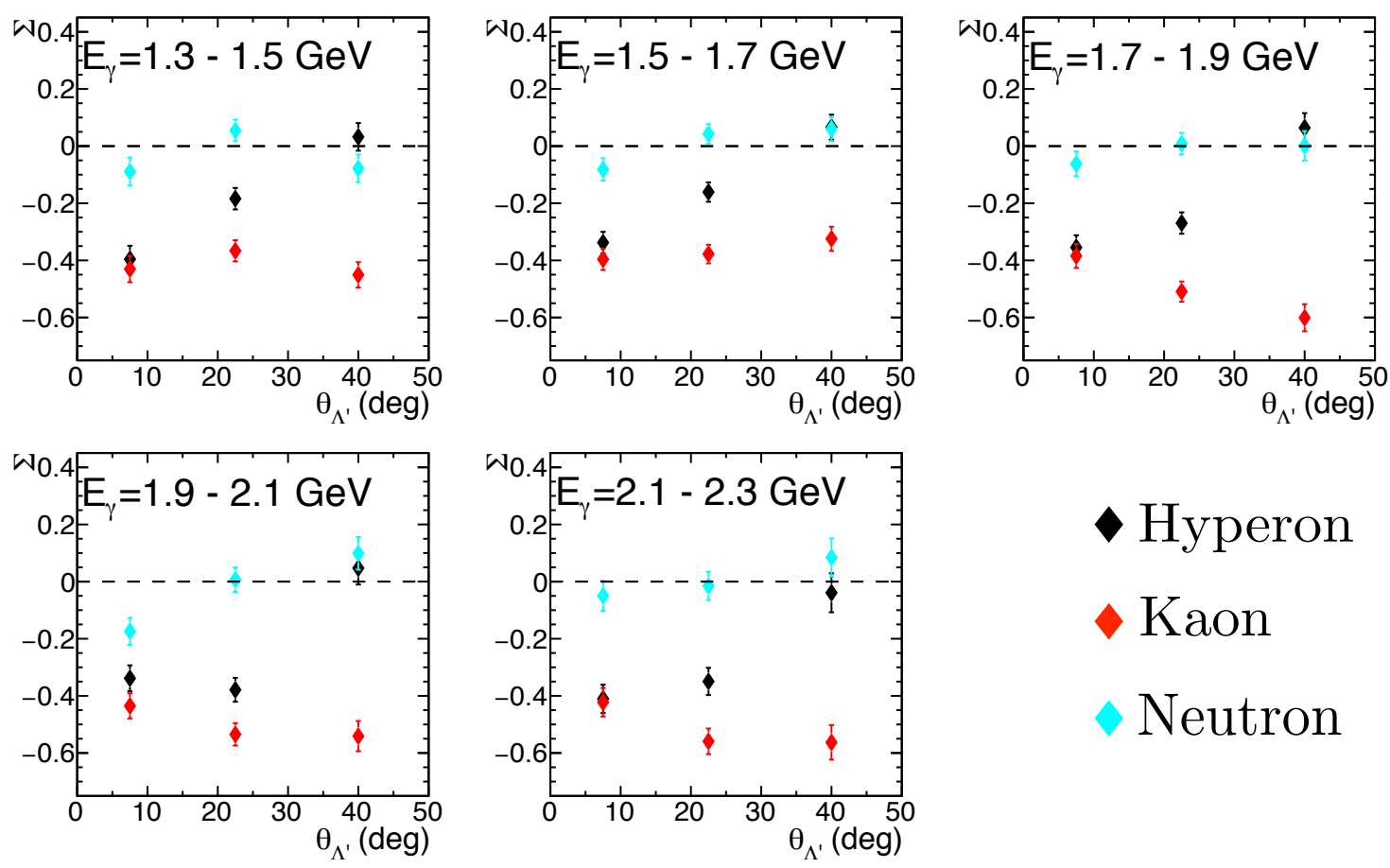

\section{- Hyperon \\ $\checkmark$ Kaon \\ Neutron}

Figure 4: Preliminary two-fold differential estimates of the beam-spin asymmetries $\Sigma_{n}$ (cyan), $\Sigma_{K}$ (red), and $\Sigma_{\Lambda}$ (black) as a function of $\theta_{\Lambda}^{\prime}$ for various photon-energy bins. Only statistical uncertainties are shown. In general, the three observables do not have to be consistent with each other. However, at large $\theta_{\Lambda}^{\prime}, \Sigma_{\Lambda}$ and $\Sigma_{n}$ are consistent within their statistical uncertainties, which suggests that the hyperon and the nucleon were produced in the same vertex, such as in $\Lambda n$ scattering. At small $\theta_{\Lambda}^{\prime}, \Sigma_{\Lambda}$ and $\Sigma_{K}$ are consistent within their statistical uncertainties, which suggests that the hyperon and the kaon were produced in the same vertex, such as in a pion-mediated mechanism. These observations are indicative of dominance of the $\Lambda n$ rescattering mechanism at large $\theta_{\Lambda}^{\prime}$ and of dominance of the pion-dominated mechanism at small $\theta_{\Lambda}^{\prime}$.

cially when the $K^{+}$and $\Lambda$ are produced in the second step through the pion-mediated mechanism. In this case, the expected values of $C_{x}$ and $C_{z}$, in a proper reference frame, are zero since the pion beam is unpolarized. If at any kinematics $C_{x}$ and $C_{z}$ for FSI are both consistent with $C_{x}$ and $C_{z}$ for $\mathrm{QF}$ production, this would indicate that kaon rescattering dominates at that kinematics. Our preliminary studies do not show any evidence that this FSI mechanism dominates our data sample in any part of the phase space.

Finally, in Fig. 7 we show preliminary estimates of four-fold differential $C_{x}$ and $C_{z}$ for finalstate interactions. The figure shows that the expected statistical uncertainties of four-fold differential observables are reasonable for the $Y N$ study, especially given the fact that the full set of observables will be simultaneously fitted. The right pannel of Fig. 7 shows the corresponding model predictions for $C_{x}$ and $C_{z}$ in a similar kinematic bin, obtained with two different $Y N$ potentials, NSC97f [15] and NSC89 [16], for the $\Lambda$ rescattering mechanism. While, both potentials reproduce the hypertriton binding energy, they lead to different predictions for $C_{x}$ and $C_{z}$. The data and the model predictions are shown in the same figure only to demonstrate the statistical uncertainties of the data and the predicted sensitivity to different $Y N$ potentials. The data and the model 

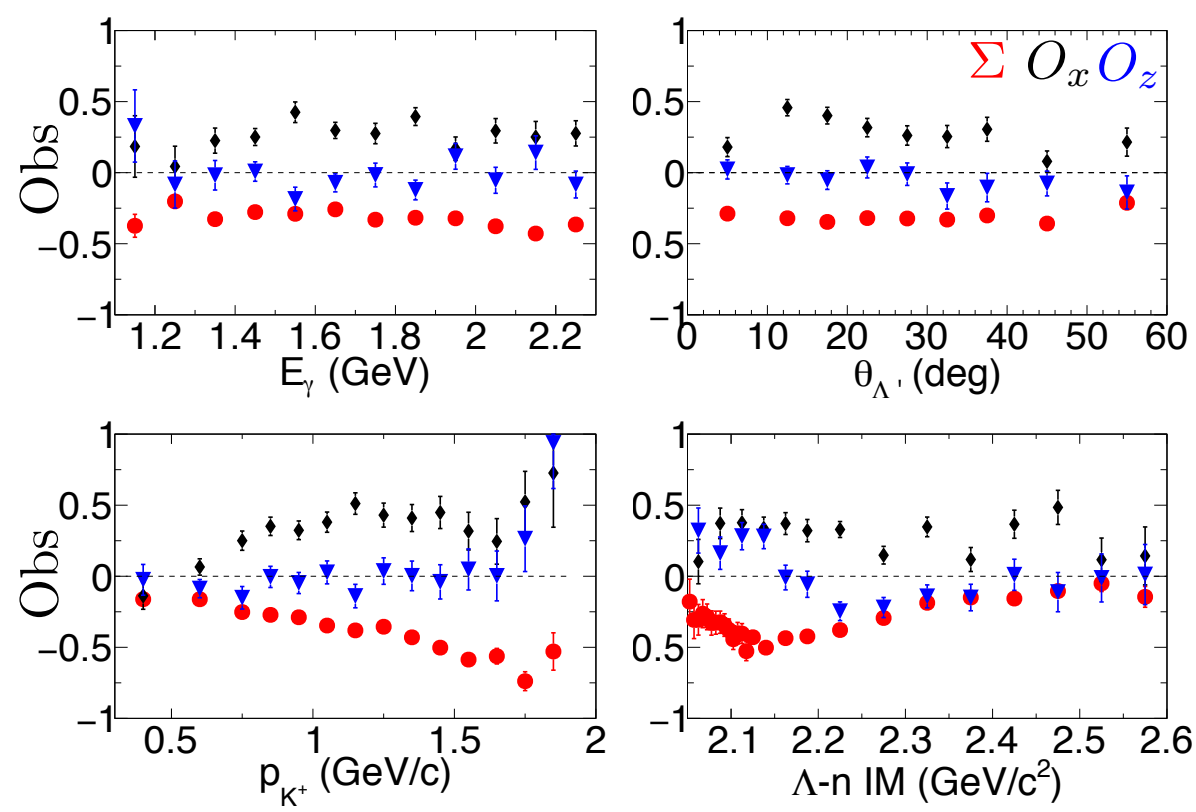

Figure 5: Preliminary one-fold differential estimates of $\Sigma_{K}, O_{z}$, and $O_{x}$ as functions of $E_{\gamma}$ (top left), $\theta_{\Lambda}^{\prime}$ (top right), $p_{K}$ (bottom left), and $I M_{\Lambda n}$ (bottom right). The observables are integrated over all other kinematic variables. Only statistical uncertainties are shown. The $\Sigma_{K}$ is binned very finely in $I M_{\Lambda n}$ in the range below the $\Sigma n$ threshold to demonstrate the statistical uncertainties of the data in this range and the potential to apply the method of [10] to extract an estimate of a spin-averaged $\Lambda n$ scattering length.
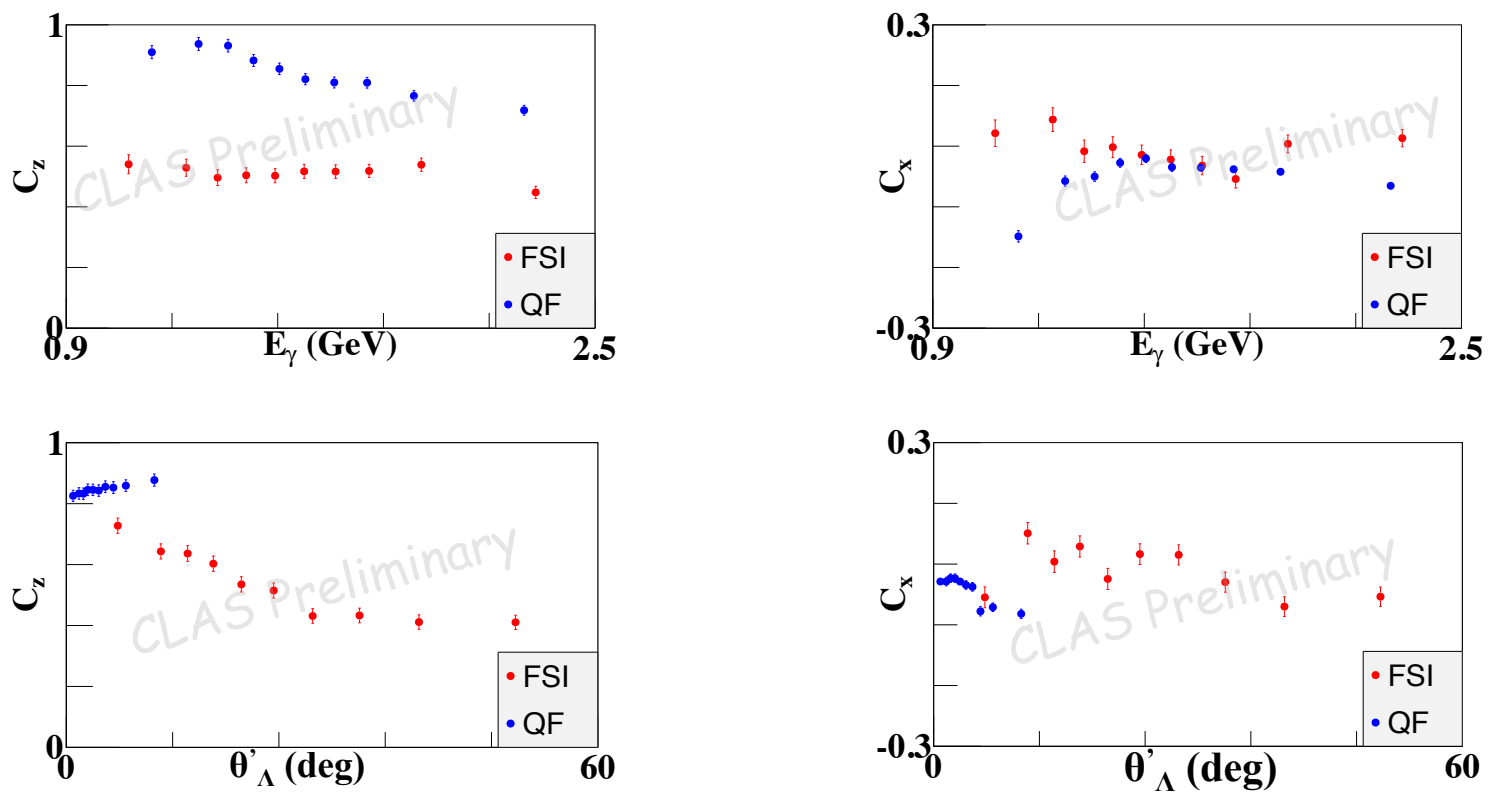

Figure 6: Preliminary one-fold differential estimates for observables $C_{x}$ and $C_{z}$ for quasi-free (QF) production and FSI as functions of $E_{\gamma}$ and $\theta_{\Lambda}^{\prime}$. One can clearly see a sizable effect of FSI. This effect is much stronger for $C_{z}$, which is close to +1 for $\mathrm{QF}$ production, than for $C_{x}$, which is very small for QF production. Only statistical uncertainties are shown. 

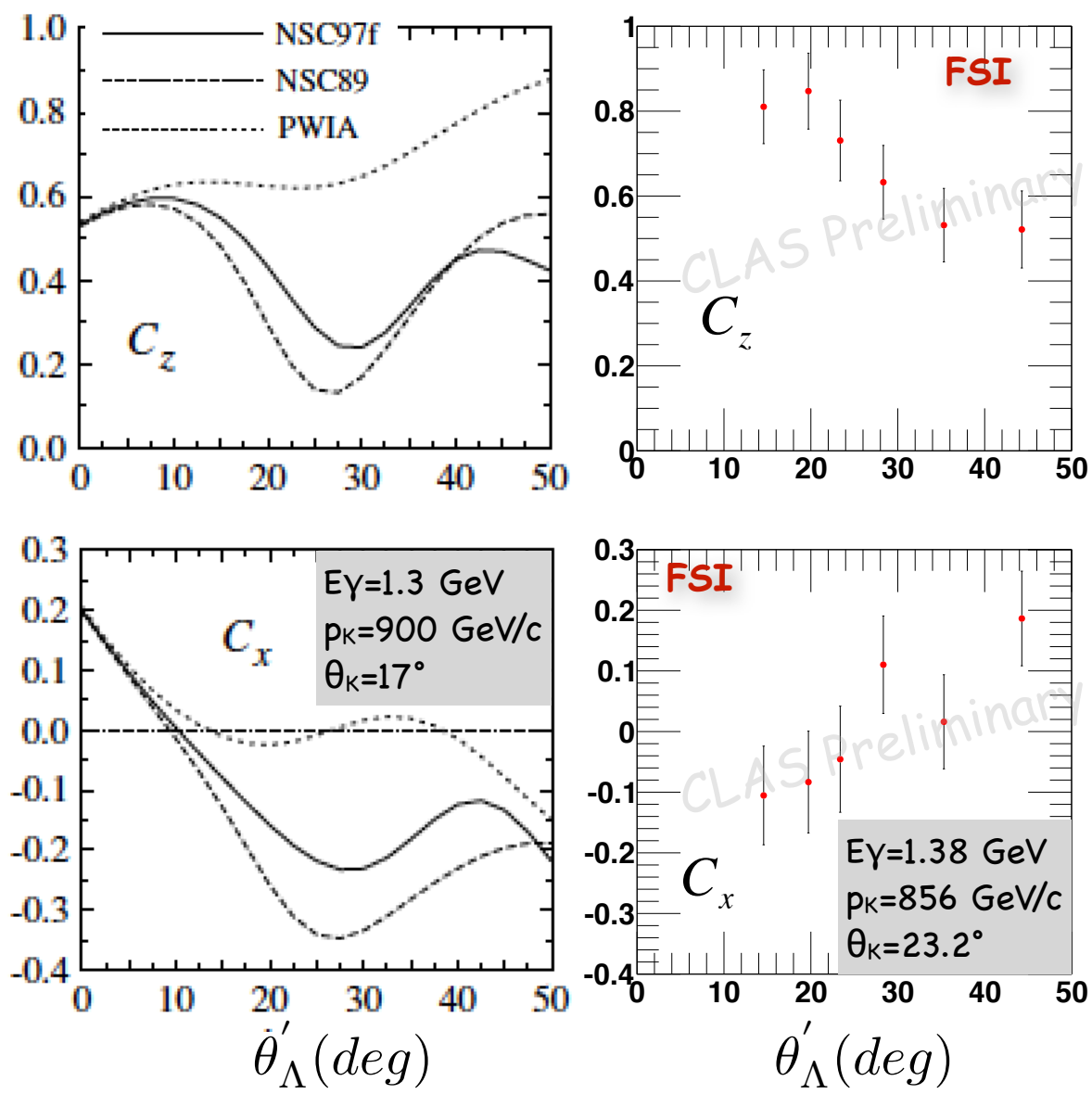

Figure 7: Right: Preliminary four-fold differential estimates of observables $C_{x}$ and $C_{z}$ for FSI. The observables are shown as a function of $\theta_{\Lambda}^{\prime}$ for the kinematic bin $\left(E_{\gamma}=1.38 \mathrm{GeV}, p_{K}=856 \mathrm{GeV} / c, \theta_{K}=23.2^{\circ}\right)$. Only statistical uncertainties are shown. Left: Model predictions of [6] for the observables in a very similar kinematic bin. The dotted curve shows predictions for QF only. The solid and dashed curves show predictions for QF and $\Lambda$ rescattering with NSC97f and NSC89 YN potentials, respectively. The data and the model predictions are plotted in the same figure only to demonstrate the statistical uncertainties of the data and the predicted sensitivity to different $Y N$ potentials. Direct comparison should not be done (see text).

predictions cannot be directly compared at this time for several reasons. First, the model only takes into account $\mathrm{QF}$ and $\Lambda$ rescattering mechanisms. Second, the elementary operator for $\gamma p \rightarrow K^{+} \Lambda$ in the model needs to be updated. Third, the kinematics of the data and the model predictions is not exactly the same, especially in terms of $\theta_{K}$. Last, the CLAS results are integrated over the $5^{\text {th }}$ kinematic variable, and thus potentially carry acceptance effects. The latter challenge is the most complex to deal with in the interpretation of our data, although several methods are available. Ultimately, the polarization observables for FSI in the reaction $\vec{\gamma} d \rightarrow K^{+} \vec{\Lambda} n$ will be included in the global analysis of $Y N$ scattering and hypernuclear data.

In summary, we have presented preliminary estimates for the observables $\Sigma, O_{x}, O_{z}, C_{x}$, and $C_{z}$ for FSI in the reaction $\vec{\gamma} d \rightarrow K^{+} \vec{\Lambda} n$. One- to four-fold differential estimates are extracted with reasonable statistical uncertainties. The results for $\Sigma$ indicate that $\Lambda n$ scattering may be dominant 
at large $\theta_{\Lambda}^{\prime}$. More detailed study of various FSI will be done by using the results for $O_{x}, O_{z}, C_{x}$, and $C_{z}$. The beam-spin asymmetry can be binned sufficiently fine at low $I M_{\Lambda n}$ to be suitable for the extraction of a spin-averaged $\Lambda n$ scattering length. Work is in progress to estimate the systematic uncertainties of the observables and to establish reliable methods for their physics interpretation. Overall, our data have sufficient coverage and statistical significance to impact the studies of the $Y N$ interaction.

\section{References}

[1] B.O. Kerbikov, B.L.G. Bekker, and R. Daling, Nucl. Phys. A 480, 585 (1988).

[2] R.A. Adelseck and L.E. Wright, Phys. Rev. C 39, 580 (1989).

[3] X. Li and L.E. Wright, Nucl. Part. Phys. 17, 1127 (1991) and references therein.

[4] H. Yamamura et al., Phys. Rev. C 61, 014001 (1999).

[5] O.V. Maxwell, Phys. Rev. C 69, 0346705 (2004).

[6] K. Miyagawa et al., Phys. Rev. C 74, 034002 (2006).

[7] A. Salam et al., Phys. Rev. C 74, 044004 (2006).

[8] A. Salam and H. Arenhövel, Phys. Rev. C 70, 044008 (2004).

[9] J.M.-Laget, Phys. Rev. C 75, 014002 (2007).

[10] A. Gasparian et al., Eur. Phys. J. A 32, 61 (2007).

[11] A. Gasparian et al., Phys. Rev. C 69, 034006 (2004).

[12] P. N-Turonski, B.L. Berman, Y. Ilieva, D. Ireland, and A. Tkabladze, Kaon Production on the Deuteron Using Polarized Photons, JLab experiment E06-103 (2006).

[13] D.I. Sober et al., Nucl. Instrum. Methods Phys. Res. A 440, 263 (2000).

[14] B. Mecking et al., Nucl. Instrum. Methods Phys. Res. A 503, 513 (2003).

[15] Th.A. Rijken, V.G.J. Stoks, and Y. Yamamoto, Phys. Rev. C 59, 21 (1999).

[16] P.M.M. Maessen, Th.A. Rijken, and J.J. de Swart, Phys. Rev. C 40, 2226 (1989). 